\title{
BS13
}

\section{Broadband Seismic Experience from Land Data in Kuwait}

\author{
A. Abdullah* (Kuwait Oil Company), A.H. El-Emam (Kuwait Oil Company (K. \\ S.C.)) \& A. Al-Sabaa (WesternGeco)
}

\section{SUMMARY}

The use of seismic data has progressed from structure interpretation to a more wide range of high-end applications including reservoir properties and geo-steering. The generation of high resolution mechanical earth models is enabled by high density, full azimuth, and broad band seismic acquisition combined with the latest data processing technologies.

Developments in acquisition and processing philosophy over the years have helped Kuwait Oil Company exploit the full potential of the acquired seismic datasets. Single-sensor technology in particular has evolved in parallel with the industry moving towards broadband, full-azimuth and high-density designs as the ideal standard. Complementary, the developments in source technology for lower frequencies have significantly helped the imaging and extracting attributes even at deeper levels (Jurassic / Paleozoic).

The high resolution single sensor seismic shall continue to provide guidance for drilling campaigns and allow for accurate identification of bypassed oil and gas, hence drilling fewer dry wells and positioning wells more accurately which in turn will directly improve the accuracy of the company's reserve estimates and the cost of producing hydrocarbon in Kuwait

In this presentation we share our broad band seismic experience and show how it led to effectively sample and remove the noise, while preserving signal fidelity and consequently how this improved seismic formed the bases to overcome previous geophysical challenges 
The use of seismic data has progressed from structure interpretation to a more wide range of high-end applications including reservoir properties and geo-steering. The generation of high resolution mechanical earth models is enabled by high density, full azimuth, and broad band seismic acquisition combined with the latest data processing technologies.

Developments in acquisition and processing philosophy over the years have helped Kuwait Oil Company exploit the full potential of the acquired seismic datasets. Single-sensor technology in particular has evolved in parallel with the industry moving towards broadband, full-azimuth and highdensity designs as the ideal standard. Complementary, the developments in source technology for lower frequencies have significantly helped the imaging and extracting attributes even at deeper levels (Jurassic / Paleozoic).

The high resolution single sensor seismic shall continue to provide guidance for drilling campaigns and allow for accurate identification of bypassed oil and gas, hence drilling fewer dry wells and positioning wells more accurately which in turn will directly improve the accuracy of the company's reserve estimates and the cost of producing hydrocarbon in Kuwait

In this presentation we share our broad band seismic experience and show how it led to effectively sample and remove the noise, while preserving signal fidelity and consequently how this improved seismic formed the bases to overcome previous geophysical challenges 